\title{
Autostabilizing airframe articulation: Animal inspired air vehicle control
}

\author{
Jonathan P. Dyhr, Noah J. Cowan, David J. Colmenares, Kristi A. Morgansen and Thomas L. Daniel
}

\begin{abstract}
The sparse sensing and limited articulation that are characteristic of human-engineered robotic systems contrast dramatically with sensorimotor systems observed in nature. Animals are richly imbued with sensors, have many points of articulation and are heavily over-actuated. In fact, the compliant nature of the body (or Plant) of most animals requires constant control input to the muscles for postural maintenance. In this study, we show how flying insects use a compliant airframe to maintain flight stability via active articulation of the frame. We first derive the equations of motion for a model flying insect, inspired by the hawkmoth, a large fast flying and agile insect. By linearizing the equations of motion about a hovering equilibrium, we demonstrate that abdominal motions are sufficient to stabilize flight on a scale of $50 \mathrm{~ms}$. We then tested whether these insects use the abdomen for flight control by first measuring the open-loop transfer function between visual pitch rotations and abdominal movement in a tethered moth preparation. The measured transfer function was consistent with an abdominal control strategy. We then closed the loop and found that moths actively stabilize visual pitch rotations using abdominal motion as the only control input. The behavior was robust to variations in gain and to a variety of visual stimuli. These experiments establish airframe articulation as a plausible control mechanism for active flight.
\end{abstract}

\section{INTRODUCTION}

Animal flight has served as inspiration for design concepts for a host of air vehicles. From the classic observations of da Vinci [1], the first to show that the center of mass and center of pressure may not align in flying animals, to exciting recent studies of wing morphing [2], compliant wing dynamics [3], [4], autostabilization in yaw [5] and a host of sensory systems in flight control such as optic flow [6] and biological gyroscopes [7], [8] we have seen the development of novel designs employed in engineered systems [9], [10]. Interestingly nearly all attention has focused on either the sensory information flow provided to flying animals, or on the kinematics of their wings and the unique aerodynamic processes that result from flapping flight. Relatively little attention has focused on how the airframe itself may play an important role in flight control. Evidence in the biological literature suggests that there is extensive neural control of body posture during flight. From the dynamic motions of

The work and experimental systems were supported by an ONR grant No. N000141010952 for KAM and TLD, NSF postdoctoral fellowship No. 1103768 for JPD, NSF grant No. 0845749 for NJC, a Mary Gates Undergraduate Research Fellowship for DJC, the Air Force Research Laboratory as part of the DARPA HI-MEMS program (TLD and J. Voldman), and a Komen Endowed Chair to TLD.

J. P. Dyhr, D. Colmenares, K.A. Morgansen and T.L. Daniel are with the University of Washington, Seattle, WA 98195 USA Email: \{jdyhr, djc26, morgansn,danielt\}@uw.edu.

N. J. Cowan, is with the Department of Mechanical Engineering, Johns Hopkins University, Baltimore, MD 21218 USA. Email: ncowan@jhu.edu. bird tails as aerodynamic control surfaces [11], [12] to even lizard tails as inertial controllers of descent paths [13] to the powerful movements of the abdomen of insects [14], [15], we see a breathtaking range of airframe motions in response to perturbations. Indeed, the promise of airframe deformation was recently explored as a method for remotely controlling insect flight paths by stimulating neural centers controlling flexion of the insect's abdomen during free flight [16].

Given the widespread occurrence of airframe deformation in biology, we sought to use this characteristic as inspiration for the design of a new generation of micro-air vehicle controllers. We use as inspiration the abdominal flexion of the flying hawkmoth to explore the control potential and stability of air vehicles with deforming airframes. We combine a dynamical systems model of the deformable airframe along with a control theoretic model to explore the stability of this system. In addition, we use real moths in miniature flight simulators to extract the transfer function for airframe deformation in response to visual pitch stimuli. Combining plant dynamic models of airframe deformation with experimentally derived sensory-response transfer functions, we are able to show that this biologically inspired approach can provide pitch stability. Further, we are able to show, via closed-loop measures of hawkmoths in the flight simulator, that their abdomen does indeed provide control authority.

The organization of the paper is as follows. A controltheoretic system model is presented in Section II. The linearized model is validated relative to live animal data in Section III. Closed-loop system response is discussed in Section IV, and conclusions are discussed in Section V.

\section{MODELING DEFORMING AIRFRAMES}

\section{A. Model setup}

We have developed an analytic model inspired by the dynamics of the flying hawkmoth, Manduca sexta [17]. Our model treats the animal as a two section airframe that can be deformed in response to a pitch stimulus. That deformation is driven by a torque at the joint between the front (thorax and head, mass $m_{t}$ ) and rear (abdomen, mass $m_{a}$ ) sections of the airframe (see Fig. 1). In addition, we assume that there is a constant lift and thrust vector $F_{w}$ that remains fixed relative to the thorax of the animal.

To determine the stability of the system we first derived the nonlinear Euler-Lagrange equations of the system in the center of mass reference frame, which can be expressed for 


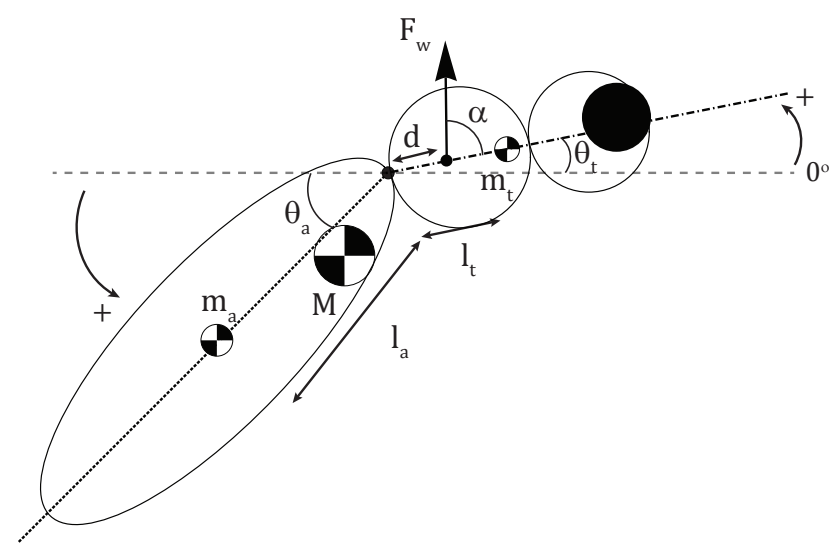

Fig. 1. The moth inspired deformable airframe consisted of a front (thorax and head) and back (abdomen) attached by a hinge joint. The center of masses of each segment $\left(m_{a}\right.$ and $\left.m_{a}\right)$ were located at fixed lengths ( $l_{a}$ and $l_{t}$ ) from the hinge. Wing forces $F_{w}$ were modeled as a constant force vector originating from the thorax at a distance $d$ from the joint oriented at a fixed angle $\alpha$ relative to the thoracic angle $\left(\theta_{t}\right)$. The thoracic and abdominal angles were changed by a torque $\tau$ applied at the joint.

kinematic variables $q=\left(x, y, \theta_{a}, \theta_{t}\right)$ as

$$
M(q) \ddot{q}+C(q, \dot{q})+N(q)=\tau,
$$

where $M(q)$ is the mass matrix, $C(q, \dot{q})$ includes Coriolis terms, $N(q)$ includes first order terms due to gravity, and $\tau$ is the torque applied at the thorax-abdomen joint. Deriving the equations of motion for the system in Fig. 1, prior to introducing constraints and external forces, yields the following:

$$
M=\left[\begin{array}{cccc}
m_{a}+m_{t} & 0 & 0 & 0 \\
0 & m_{a}+m_{t} & 0 & 0 \\
0 & 0 & \alpha_{11} & \alpha_{12} \\
0 & 0 & \alpha_{12} & \alpha_{22}
\end{array}\right]
$$

where $\alpha_{11}=\frac{l_{a}^{2} m_{a} m_{t}+I_{a}\left(m_{a}+m_{t}\right)}{m_{a}+m_{t}}, \alpha_{12}=\frac{l_{a} l_{t} m_{a} m_{t} \cos \left(\theta_{a}-\theta_{t}\right)}{m_{a}+m_{t}}, \alpha_{22}=$ $\frac{l_{t}^{2} m_{a} m_{t}+I_{t}\left(m_{a}+m_{t}\right)}{m_{a}+m_{t}}$, and

$$
C+N=\left[\begin{array}{c}
0 \\
g\left(m_{a}+m_{t}\right) \\
\frac{l_{a} l_{t} m_{a} m_{t} \sin \left(\theta_{a}-\theta_{t}\right) \dot{\theta}_{t}^{2}}{m_{a}+m_{t}} \\
-\frac{l_{a} l_{t} m_{a} m_{t} \sin \left(\theta_{a}-\theta_{t}\right) \dot{\theta}_{a}^{2}}{m_{a}+m_{t}}
\end{array}\right] .
$$

The wing lift forces $F_{w}$, fixed at $90^{\circ}$ relative to the major axis of the thorax, were introduced as constraints on the term $N$. Equation (1) can be expressed as four first-order equations by replacing $q$ with state vector $z$ :

$$
z=\left[\begin{array}{l}
z_{1} \\
z_{2}
\end{array}\right]
$$

where

$$
z_{1}=\left[\begin{array}{c}
x \\
y \\
\theta_{a} \\
\theta_{t}
\end{array}\right], \quad z_{2}=\left[\begin{array}{c}
\dot{x} \\
\dot{y} \\
\dot{\theta}_{a} \\
\dot{\theta}_{t}
\end{array}\right]
$$

TABLE I

MODEL PARAMETERS

\begin{tabular}{|c|c|c|}
\hline$m_{a}$ & $l_{a}$ & $I_{a}$ \\
\hline $0.999 g$ & $0.0153 m$ & $2.9 \times 10^{-4} \mathrm{~g} \cdot \mathrm{m}^{2}$ \\
\hline \hline$m_{t}$ & $l_{t}$ & $I_{t}$ \\
\hline $0.832 g$ & $0.008 m$ & $0.58 \times 10^{-4} \mathrm{~g} \cdot \mathrm{m}^{2}$ \\
\hline \hline$\alpha$ & $d$ & $F_{w}$ \\
\hline $90^{\circ}$ & $0.01 \mathrm{~m}$ & $1.831 \mathrm{~g} \cdot 9.8 \frac{\mathrm{m}}{s^{2}}$ \\
\hline
\end{tabular}

Because $\dot{z}_{1}=z_{2}$, the Lagrange equations become

$$
\dot{z}=f(z, u)=\left[\begin{array}{c}
z_{2} \\
M\left(z_{1}\right)^{-1}\left(\tau-C\left(z_{1}, z_{2}\right)-N\left(z_{1}\right)\right)
\end{array}\right] .
$$

The torque $\tau$ acts on the abdominal and thoracic angles with equal, but opposite, magnitudes. For a real moth, the abdominal-thoracic joint does not freely rotate, but rather has some inherent dynamic properties. We model the joint as a torsional spring-damped system, with spring constant $k$ and coefficient of damping $b$. The abdomen is also assumed to have a natural rest angle $\theta_{0}$, determined by finding the angle at which the spring and gravitational constants balanced so that the center of mass was located directly under the center of lift. In addition to the passive dynamic properties of the joint, the moth actively manipulates abdominal angle via muscle actuation, which we include as a control torque $u_{1}$. The net torque at the joint is then

$$
\tau=\left[\begin{array}{c}
0 \\
0 \\
u_{1}-k\left(-\theta_{0}+\theta_{a}-\theta_{t}\right)-b\left(\dot{\theta}_{a}-\dot{\theta}_{t}\right) \\
-u_{1}+k\left(-\theta_{0}+\theta_{a}-\theta_{t}\right)-b\left(\dot{\theta}_{a}-\dot{\theta}_{t}\right)
\end{array}\right] \cdot z_{1}^{T} .
$$

These equations constitute the complete non-linear dynamics of the model moth. The final step for evaluating the model was to input real parameter values. To simplify the stability analysis, we evaluated the model in the equilibrium state of hovering, permitting a linear approximation of the full nonlinear equations of motion. Prior to linearizing the model, we also dropped states that were of minimal interest to our analysis $(x, y, \dot{y})$.

\section{B. Parameters}

The masses of all segments and their moments of inertia follow from previously published data and are summarized in Table I [18]. In addition, the values of $k$ and $b$ were determined from perturbation experiments done on animals during tethered flight. In these experiments a physical impulse was applied to the tip of the moth abdomen during tethered flight while measuring the change in abdominal angle (Fig. 2). After the peak in the abdominal angle, due to the perturbation, moths quickly recover to within 5 degrees of the initial position with minimal oscillation. These results suggest that the abdomen behaves as a critically damped system during flight. Furthermore, the quick recovery to the original position suggested that the abdominal angle was effectively specified by a positional command (stiffness and damping were both large). Thus, the hinge dynamics need 
not be included in the model, as whatever command control is being sent to the abdomen is effectively converted to an angular position.

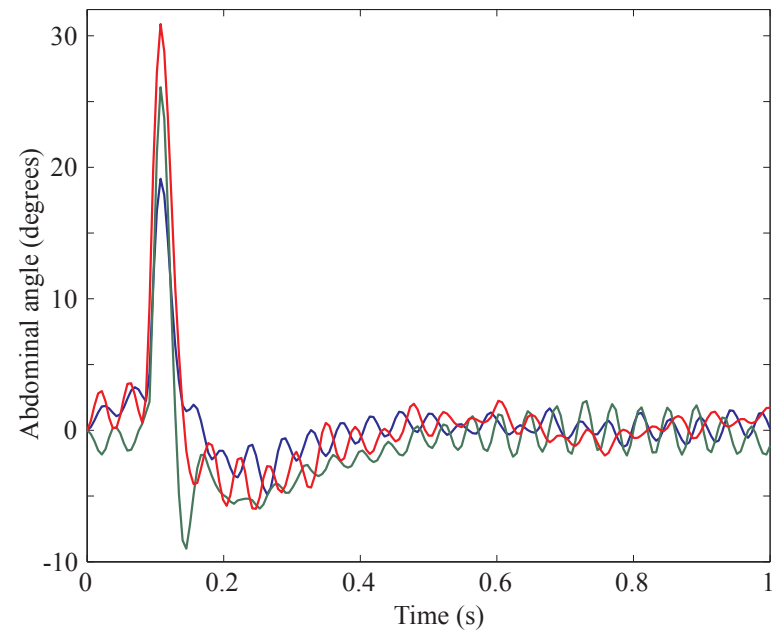

Fig. 2. Moths quickly recover from abdominal perturbations during tethered flight. The average angular deflections for three moths (colored traces) in response to a physical impulse (peak deflection) show that the abdominalthoracic joint is critically damped during flight. The small $20 \mathrm{~Hz}$ oscillations are due to the wing strokes of the moth

\section{Transfer function}

Armed with model parameters, and our ability to equate torque control to angular position of the abdomen, we model the control input as an angular control variable $u$ such that:

$$
u_{1}=k u \text {. }
$$

Both $b$ and $k$ were assumed to be very large, resulting in a very strong restoring force with minimal temporal dynamics. Hence, the spring constant effectively converts the angle command $u$ into the torque required to achieve a specific abdominal-thoracic angle. The linearized equations yielded the following transfer function from the input $u$ (proportional to $\theta_{a}-\theta_{t}$ ) to the thoracic measurement(pitch angle) in Laplace space

$$
P(s)=K_{m} \frac{s^{2}+z_{m}^{2}}{s^{2}},
$$

where $K_{m}=-0.69$ and $z_{m}^{2}=225 s^{-1}$. This transfer function can also be expressed as an ordinary differential equation:

$$
\ddot{\theta}_{t}(t)=K_{m}\left(\ddot{u}(t)+z_{m}^{2} u(t)\right) .
$$

Equation (6) has two temporal dynamics. One is on the time scale of the position change of the abdomen relative to the thorax, redirecting the lift vector relative to the center of mass. The other time scale is associated with the rotation of the body as whole in response to that redirection of force.

\section{TRANSFER FUNCTIONS FROM LIVING ANIMALS: MODEL VALIDATION}

A. Extracting transfer functions from living animals: methods and results

To determine the transfer function used by the animal during flight, we constructed an insect-scaled flight simu-

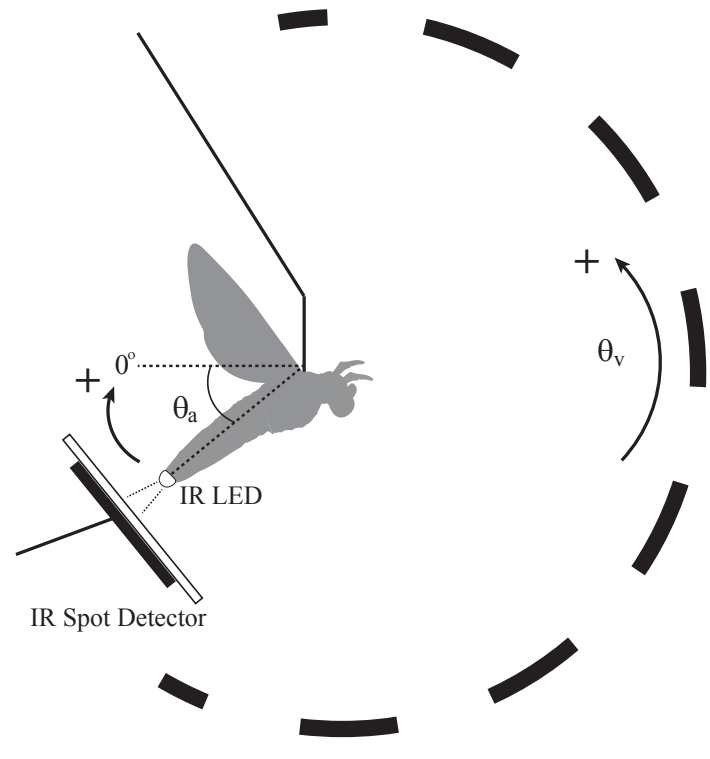

Fig. 3. Moths were tethered in a miniature flight simulator and presented with visual stimuli rotating about the pitch axis (dashed circle, $\theta_{v}$ ). Abdominal angle, $\theta_{a}$, was measured using two methods. For open-loop experiments, the angle was measured from the side using a high-speed camera. Real-time measurement of abdominal angle for closed-loop experiments was tracked using an IR spot detector and an IR LED glued to the tip of the abdomen.

lator which produced visual pitch stimuli that drive neural reflexive abdominal flexions (see Fig. 3 and [14], [19]). Tethered animals were placed in the flight simulator and exposed to an open-loop stimulus that consisted of a pattern of horizontally oriented dark and light bars with a spatial frequency of 0.03 cycles/degree (consistent with a visual stimulus that has maximal response [20]). That pattern was moved in the pitch direction with a temporally varying pitch velocity that was composed of a sum of sinusoidal motions. That sum of signs consisted of 20 prime multiple frequencies that were logarithmically spaced between 0.1 and $20 \mathrm{~Hz}$ (see Fig. 4b and [21]). The position of the abdomen was tracked during these trials using a high-speed video camera (Basler Pilot GigE, Basler Vision Technologies, Ahrensburg, Germany) operating at 186 frames per second. Abdominal angle data was digitized from the images with custom MATLAB software (DLTdataviewer; [22]).

From the data summarized in Fig. 4, we calculated the gain and phase of the abdominal flexion in response to the visual pitch stimulus. These then were used to establish the transfer function which shows that abdominal responses were strongly attenuated at frequencies below $1 \mathrm{~Hz}$, after which they plateau as frequency increases.

The phase plot (Fig. 5) shows a pronounced, and approximately linear, roll-off indicative of a fixed time delay. Together, these data suggest that the input-output relationship is consistent with a transfer function consisting of a high-pass filter and a fixed time delay. We estimate these responses to have a gain $K=0.44$, one zero $z=0.08$, one pole $p=0.75$, and a time delay $\tau_{d}=0.042 s$. In the Laplace domain, the 

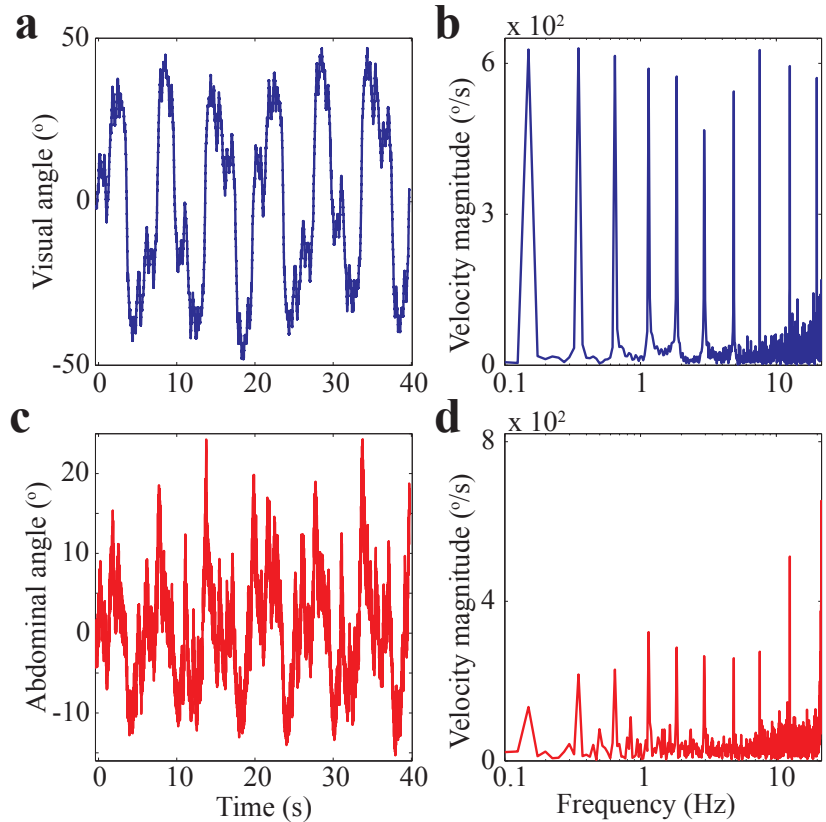

Fig. 4. Example stimulus (top, blue) and abdominal response (bottom, red) data are shown in both the time domain (left) and frequency domain (right) for an open-loop sum of sines trial. Stimuli were normalized in velocity amplitude. Behavioral response frequencies were precisely matched to those present in the visual stimulus.

function has the form

$$
G(s)=K \frac{s+z}{s+p} \cdot e^{-s \tau_{d}} .
$$

\section{B. Observed and predicted transfer functions shows stabi- lizing responses}

The above experiments provided two transfer functions, one for the open-loop tethered flight experiments and a second for a free flight model derived from first principles. These transfer functions correspond to the Sensor/Controller blocks and Plant block in the full feedback system respectively. For a linear system, we can easily determine the closed-loop dynamics $(H(s))$ of the system by multiplying them in the Laplace domain:

$$
H(s)=\frac{G(s) \cdot P(s)}{1+G(s) \cdot P(s)} .
$$

In order to determine the stability of this time-delayed system, a Nyquist plot has been constructed (Fig. 6). For a given open-loop transfer function, the resulting closed-loop system is stable when the minus one point on the real axis is not encircled. For the open-loop system $G(s) \cdot P(s)$, we see that the system does not encircle the minus one point and is stable. However, the system is very close to marginal stability, and a slight increase in the gain is sufficient to destabilize the system. Including other relevant factors, such as the inertial dynamics of wings, has the potential to further stabilize, or slightly destabilize, the system.
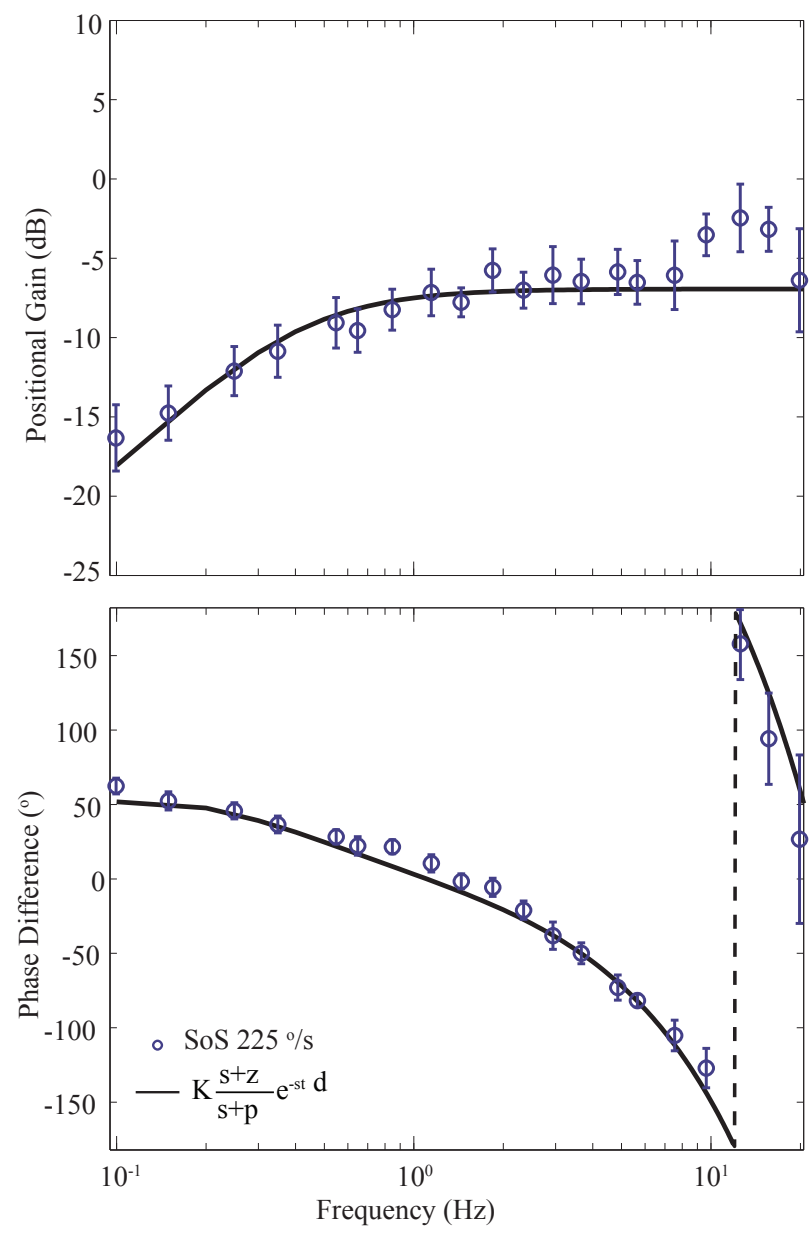

Fig. 5. Experimentally measured gain and phase data from 7 moths was averaged (error bars indicate 95\% confidence intervals) and approximated by a first order high-pass filter with a fixed time delay (black line; see text for details).

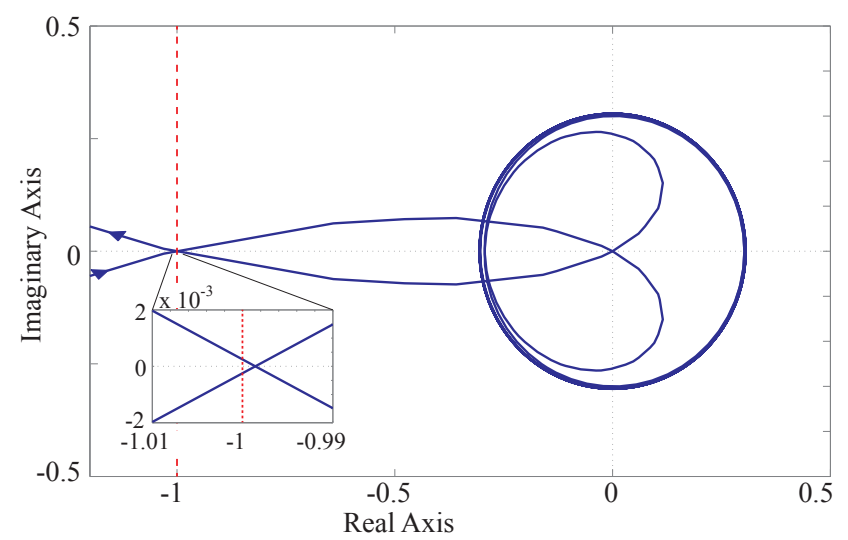

Fig. 6. The Nyquist plot reveals that airframe deformations contribute to flight stability. The combined experimental (Controller) and model (Plant) transfer functions are stable, but very close to the stability margin. 


\section{CONTROL AUthority OF THE AIRFRAME: CLOSED-LOOP RESPONSES}

The transfer function estimated from the arena experiments is the open-loop response of a tethered moth. The dynamics of the sensory input, controller output and plant dynamics will be different for a moth with either closed-loop control of the visual stimulus, free flight body dynamics, or both. While we have established the sufficiency of abdominal flexion as a stabilizing feature of flight control, we have not shown it has control authority in the more challenging closed-loop context. Accordingly, we modified the flight simulator to have real time position sensing of the abdomen as an input into the visual pitch stimulus. The stimulus consisted of a horizontal bar drifting in the pitch upward direction at a velocity of approximately 16.5 degrees per second. The position of the abdominal tip, tracked with an IR LED and an IR 2D spot detector (UDT Technology), was used to offset the motion of the visual pitch stimulus at a velocity that was directly proportional to the abdominal angle (relative to a rest position).

Results show that a tethered moth can indeed control its visual horizon using its abdomen (Fig. 7). The abdomen is held by the animal at an angular deflection that offsets the drift of the visual stimulus. Episodically, the animal "gives up," and we see the inherent drift signal of the visual stimulus. The animal, however, can abdominally "grab" the signal by moving the abdomen to counteract the drift and once again stabilize its visual world. Furthermore, this behavior was robust to four-fold changes in gain and was stable for a variety of visual stimuli, including horizontal black bars, spatial white noise patterns, and natural images.

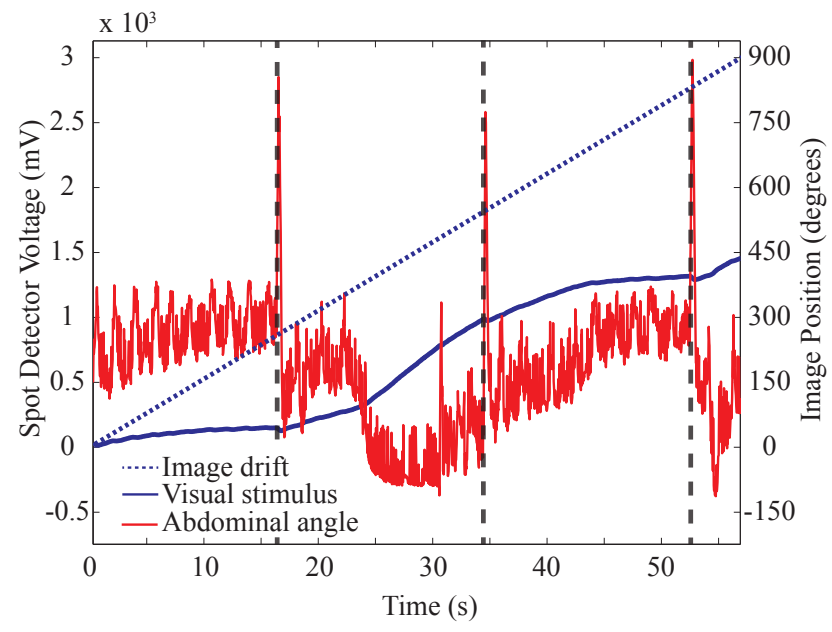

Fig. 7. Closed-loop stabilization of a visual pattern (solid blue line) based on the spot detector voltage (red line), proportional to the abdominal angle, demonstrates the control potential of the abdomen. Three trial of closedloop control are shown, demarcated by dashed lines, during which moths had closed-loop control of the visual world. The moth holds the drifting visual pattern (drift represented by the dotted blue line) in the first and third trials, but briefly loses the pattern during the second trial. Large spikes in abdominal angle are short epochs between trials where the visual stimulus was turned off. The high frequency component is the inertial reaction of the abdomen to wing beats.

\section{CONCLUSIONS}

The widespread occurrence of changes in body posture in flying animals provides inspiration for the concept of airframe deformation as a possible control mechanism in air vehicles. This effect is particularly evident in the flight control system of large insects where, in the hawkmoth, powerful flexion of the abdomen occurs in response to visual pitch stimuli. The combination of dynamic models of such deforming airframes and control theoretic analyses of these systems points to a novel mechanism for a pitch stabilizing controller. Evidence from animals in both openand closed-loop flight simulators suggests that neural control of abdominal angle in flying insects does indeed provide control authority. Taken together, these results suggest that that it is possible to implement airframe deformation in artificial systems, particularly agile micro air vehicles [23].

It is important to note that, in addition to playing a key role in actuating pitch control and changes in flight paths, deformable airframes can also play an unexplored role in sensing. This follows from another biologically inspired observation: the positions of nearly all body parts of moving animals are sensed (proprioceptively) by the nervous system in order to inform a moving creature of the relative states of stretch and position of locomotor structures. We suspect that the abdomen of large flying insects is also equipped with proprioceptive structures, providing the animal information about the angle of the abdomen. By inference, it is logical to assume that passive flexion of the abdomen may result from inertial reactions to abrupt pitch (or yaw) rotations. The proprioceptive information about angle of the abdomen may inform a flying animal about rotational motions. Thus the abdomen and its associated proprioceptive information suggest that it could serve a dual role as a sensor and an actuator. Such combined sensing and actuation systems present exciting nonlinear control theoretic challenges.

\section{ACKNOWLEDGEMENTS}

We thank Armin Hinterwirth and Amber Fechko for design assistance of the flight simulators.

\section{REFERENCES}

[1] L. da Vinci, "Codex on the flight of birds," circa 1505.

[2] D. Lentink, U. K. Müller, E. J. Stamhuis, R. de Kat, W. van Gestel, L. L. M. Veldhuis, P. Henningsson, A. Hedenström, J. J. Videler, and J. L. van Leeuwen, "How swifts control their glide performance with morphing wings," Nature, vol. 446, no. 7139, pp. 1082-1085, 2007.

[3] A. M. Mountcastle and T. L. Daniel, "Vortexlet models of flapping flexible wings show tuning for force production and control," Bioinspiration \& Biomimetics, vol. 5, no. 4, p. 045005, 2010.

[4] T. Nakata and H. Liu, "Aerodynamic performance of a hovering hawkmoth with flexible wings: a computational approach," Proceedings of the Royal Society B: Biological Sciences, vol. 279, no. 1729, pp. 722731, 2012.

[5] T. L. Hedrick, B. Cheng, and X. Deng, "Wingbeat time and the scaling of passive rotational damping in flapping flight," Science, vol. 324, no. 5924, pp. 252-255, 2009.

[6] J. P. Dyhr and C. M. Higgins, "Non-directional motion detectors can be used to mimic optic flow dependent behaviors," Biological Cybernetics, vol. 103, no. 6, pp. 433-446, 2010. 
[7] T. L. Daniel, Z. Aldworth, A. Hinterwirth, and J. Fox, "Insect inertial measurement units: Gyroscopic sensing of body rotation," in Frontiers in Sensing: Biology and Engineering, F. Barth and M. V. Srinivasan, Eds. Springer, Wien-New York: Springer-Verlag, 2012, pp. 286-297.

[8] W. P. Chan, F. Prete, and M. H. Dickinson, "Visual input to the efferent control system of a fly's "gyroscope"," Science, vol. 280, no. 5361, pp. 289-292, 1998.

[9] A. M. Hyslop and J. S. Humbert, "Autonomous navigation in ThreeDimensional urban environments using Wide-Field integration of optic flow," AIAA Journal of Guidance, Control, and Dynamics, vol. 33, no. 1, pp. 147-159, 2010.

[10] J. Conroy, G. Gremillion, B. Ranganathan, and J. S. Humbert, "Implementation of wide-field integration of optic flow for autonomous quadrotor navigation," Autonomous Robots, vol. 27, no. 3, pp. 189 198, 2009.

[11] A. L. R. Thomas, "On the aerodynamics of birds' tails," Philosophical Transactions of the Royal Society of London. Series B: Biological Sciences, vol. 340, no. 1294, pp. 361-380, 1993.

[12] A. Balmford, A. L. R. Thomas, and I. L. Jones, "Aerodynamics and the evolution of long tails in birds," Nature, vol. 361, no. 6413, pp. 628-631, 1993.

[13] T. Libby, T. Y. Moore, E. Chang-Siu, D. Li, D. J. Cohen, A. Jusufi, and R. J. Full, "Tail-assisted pitch control in lizards, robots and dinosaurs," Nature, vol. 481, no. 7380, pp. 181-184, 2012.

[14] A. J. Hinterwirth and T. L. Daniel, "Antennae in the hawkmoth manduca sexta (lepidoptera, sphingidae) mediate abdominal flexion in response to mechanical stimuli," Journal of Comparative Physiology A: Neuroethology, Sensory, Neural, and Behavioral Physiology, pp. $1-10,2010$.

[15] J. M. Camhi, "Sensory control of abdomen posture in flying locusts," Journal of Experimental Biology, vol. 52, no. 3, pp. 533-537, 1970.

[16] W. M. Tsang, Z. Aldworth, A. Stone, A. Permar, R. Levine, J. G. Hildebrannd, T. L. Daniel, A. I. Akinwande, and J. Voldman, "Insect flight control by neural stimulation of pupae-implanted flexible multisite electrodes," in The Proceeding of $\mu$ TAS 2008. San Diego, California, USA: The Chemical and Biological Microsystems Society, 2008, pp. 1922-1924.
[17] J. P. Dyhr, T. L. Daniel, K. A. Morgansen, and N. J. Cowan, "Flexible strategies for flight control: an active role for the abdomen," in review.

[18] T. L. Hedrick and T. L. Daniel, "Flight control in the hawkmoth Manduca sexta: the inverse problem of hovering," Journal of Experimental Biology, vol. 209, no. 16, pp. 3114-3130, 2006.

[19] A. S. Fechko, A. J. Hinterwirth, and T. L. Daniel, "Gaining insight: visual feedback control in the hawkmoth Manduca sexta." in Soci ety for Integrative and Comparative Biology Annual Meeting 2012, Charleston, SC, 2012.

[20] J. C. Theobald, E. J. Warrant, and D. C. O'Carroll, "Wide-field motion tuning in nocturnal hawkmoths," Proceedings of the Royal Society B: Biological Sciences, vol. 277, no. 1683, pp. 853-860, 2010.

[21] E. Roth, K. Zhuang, S. A. Stamper, E. S. Fortune, and N. J. Cowan, "Stimulus predictability mediates a switch in locomotor smooth pursuit performance for eigenmannia virescens," The Journal of Experimental Biology, vol. 214, no. 7, pp. 1170-1180, 2011.

[22] T. L. Hedrick, "Software techniques for two- and three-dimensional kinematic measurements of biological and biomimetic systems," Bioinspiration \& Biomimetics, vol. 3, no. 3, p. 034001, 2008.

[23] A. Demir, J. P. Dyhr, M. Mert Ankarali, K. A. Morgansen, T. L. Daniel, and N. J. Cowan, "Inertial redirection of thrust forces via a exible airframe," in 15th International Conference on Climbing and Walking Robots and the Support Technologies for Mobile Machines, Baltimore, MD, USA, 2012. 